\title{
Feline Bronchioloalveolar Lung
}

\section{Carcinoma}

National Cancer Institute

\section{Source}

National Cancer Institute. Feline Bronchioloalveolar Lung Carcinoma. NCI Thesaurus.

Code C134559.

Bronchioloalveolar lung carcinoma that occurs in a cat. 